Article

\title{
Comparative Performance of Thermoacoustic Heat Exchangers with Different Pore Geometries in Oscillatory Flow. Implementation of Experimental Techniques
}

\author{
Antonio Piccolo ${ }^{1, *}$ (D), Roberto Siclari ${ }^{1}$, Fabrizio Rando ${ }^{1}$ and Mauro Cannistraro ${ }^{2}$ \\ 1 Department of Engineering, University of Messina, Contrada di Dio, 98166 S. Agata (Messina), Italy; \\ robsiclari@gmail.com (R.S.); fabrizio.rando@studenti.unime.it (F.R.) \\ 2 Department of Architecture, University of Ferrara, Via della Ghiara 36, 44121 Ferrara, Italy; \\ mauro.cannistraro@unife.it \\ * Correspondence: apiccolo@unime.it; Tel.: +39-090-397-7311
}

Received: 24 July 2017; Accepted: 31 July 2017; Published: 2 August 2017

\begin{abstract}
Heat exchangers (HXs) constitute key components of thermoacoustic devices and play an important role in determining the overall engine performance. In oscillatory flow conditions, however, standard heat transfer correlations for steady flows cannot be directly applied to thermoacoustic HXs, for which reliable and univocal design criteria are still lacking. This work is concerned with the initial stage of a research aimed at studying the thermal performance of thermoacoustic HXs. The paper reports a detailed discussion of the design and fabrication of the experimental set-up, measurement methodology and test-HXs characterized by two different pore geometries, namely a circular pore geometry and a rectangular (i.e., straight fins) pore geometry. The test rig is constituted by a standing wave engine where the test HXs play the role of ambient HXs. The experiment is conceived to allow the variation of a range of testing conditions such as drive ratio, operation frequency, acoustic particle velocity, etc. The procedure for estimating the gas side heat transfer coefficient for the two involved geometries is described. Some preliminary experimental results concerning the HX with straight fins are also shown. The present research could help in achieving a deeper understanding of the heat transfer processes affecting HXs under oscillating flow regime and in developing design optimization procedures.
\end{abstract}

Keywords: thermoacoustics; heat exchangers; heat transfer; acoustic power

\section{Introduction}

This paper addresses the issues of heat transfer in heat exchangers working under oscillatory flow conditions, which are typically found in thermoacoustic devices. Thermoacoustic engines are a new class of energy conversion devices (prime movers, refrigerators and heat pumps) whose operation relies on the interaction between heat and sound in close proximity of solid surfaces, a phenomenon identified as "thermoacoustic effect" [1,2]. Since in these devices the synchronization among the compression, expansion and heat transfer phases of the thermodynamic gas cycle is naturally accomplished by an acoustic wave, a number of technological benefits directly result. First of all, there is the complete absence of moving mechanical parts (pistons, sliding seals, etc.) which leads to engineering simplicity, reliability, longevity and low maintenance costs. Secondly, they are intrinsically low cost devices, being constituted basically by a small number of standard components made of inexpensive and common materials. Furthermore, they use environment friendly working fluids, can be employed in a large variety of applications (those involving heating, cooling or power generation [3-5]) and 
can be driven by different power sources (gas/biomass combustion, solar energy, waste heat, etc.). These characteristics make thermoacoustic technology a discipline of relevant interest for the energy industry giving it a primary position among emerging renewable energy technologies.

A typical thermoacoustic device consists of (a) an acoustic network (acoustic resonator), (b) an electro-acoustic transducer, (c) a porous solid medium (namely a regenerator in travelling-wave systems [6] or a stack in standing-wave systems [7]) and (d) at least a pair of heat exchangers (HXs) [8]. The stack/regenerator is the component where the desired heat/sound energy conversion takes place. "Hot" and "Cold" HXs, placed in close proximity of both ends of the stack/regenerator, absorb or supply heat from its ends thus enabling heat communication with external heat sources and sinks.

Heat exchangers constitute, in addition to the stack/regenerator, the highly dissipative components of thermoacoustic engines. Their porous structure entails, in fact, considerable flow resistance while steep thermal gradients are generally imposed on them to sustain the required heat fluxes. When designing efficient HXs aimed at transferring a target heat load the HX length along the direction of acoustic oscillation and the pore hydraulic radius should be simultaneously optimized in order to:

- $\quad$ Provide the heat transfer surface area compatible with minimum acoustic power loss caused by thermal and viscous dissipation;

- $\quad$ Provide the temperature drop between the HX and the adjacent fluid compatible with minimum thermal irreversibility associated to heat transfer [9,10]. An optimized HX should be able to achieve high transfer rates under small temperature differences in conjunction to low acoustic dissipation.

Although thermoacoustic HXs constitute fundamental components of thermoacoustic devices their design constitute up to now a technical and engineering challenge. This is due to the fact that the flow is oscillatory and the existing knowledge for steady flow arrangements is of little practical value [11-13]. As a consequence, standard heat transfer correlations for steady flows cannot be directly applied to thermoacoustic HXs, for which reliable and unambiguous design criteria are still lacking.

This work is concerned with the initial stage of a research aimed at studying the thermal performance of thermoacoustic HXs working under oscillatory flow conditions. The paper reports a detailed discussion of the design and fabrication of the experimental set-up, measurement methodology and test-HXs characterized by two different pore geometries, namely a circular pore geometry and a rectangular (i.e., straight fins) geometry. Some preliminary experimental results concerning the HX with straight fins are also shown. The research is aimed at achieving a deeper insight into the heat transfer processes affecting HXs under oscillating flow regime. This could help in developing optimization procedures of their performance in the design phase.

\section{The Experimental Set-Up}

The test rig considered for this research is a home-made standing wave thermoacoustic engine using air at atmospheric pressure as working fluid. A schematic of the engine is shown in Figure 1. The engine comprises four basic elements, namely an acoustic resonator filled with air at atmospheric pressure, a stack, an ambient heat exchanger and an electric heater. The engine works by heating one edge of the stack by the heater while anchoring the other edge to ambient temperature by the ambient HX. Acoustic waves will spontaneously generate in the device when the temperature gradient across the stack overcomes an onset level (depending mainly on the length and position of the stack inside the resonator).

- The resonator is made of stainless steel and has a variable cross section geometry to suppress harmonic generation [14] and to match the target operation frequency $(155 \mathrm{~Hz})$ by a shorter total length compared to a straight (constant diameter) resonator. It is essentially a pressure vessel defining the working frequency of the system and the pressure-velocity phase relationship of the 
acoustic wave. The resonator comprises a "hot" duct with a diameter of $10.5 \mathrm{~cm}$ and $10 \mathrm{~cm}$ long, a stack holder with a diameter of $10.5 \mathrm{~cm}$ and $8 \mathrm{~cm}$ long and a narrow duct with a diameter of $5.3 \mathrm{~cm}$ and $20 \mathrm{~cm}$ long. The wide ducts ("hot" duct and stack holder) and the narrow duct are coupled by a conical shaped section and terminate in a buffer volume. The resonance frequency of the rig can be varied by modifying its length through additional segments.

- The stack is a honeycomb stack made of a ceramic material (Celcor) characterized by a low thermal conductivity and a high specific heat. These properties enhance the stack performance in converting heat to acoustic power [15]. It fits the stack holder and is $8 \mathrm{~cm}$ long in the direction of the resonator axis (the longitudinal direction of acoustic vibration). The pores have a squared shape and are characterized by a hydraulic radius of around $0.28 \mathrm{~mm}$. The cell density is near $400 \mathrm{cpsi}$. The stack placed inside the stack-holder and a magnified view of a portion of it are shown in Figure 2.

- The heater is fabricated by passing about $4 \mathrm{~m}$ of a Ni-Cr wire $(30 \Omega / \mathrm{m})$ uniformly through the pores of a honeycomb ceramic slice $1 \mathrm{~cm}$ long along the resonator axis (and made of the same material of the stack) that served as a support. The slice is inserted on one side of a stainless steel ring, $2 \mathrm{~cm}$ long along the resonator axis, equipped with feedthroughs for power cables. The heater is placed between the "hot" duct and the stack holder. It is fed through a variable voltage autotransformer and is able to dissipate up to $600 \mathrm{~W}$ of electric power. A picture of the heater is shown in Figure 3.

- The HXs under test (widely descripted in the next section) function in this arrangement as ambient HXs (i.e., subtract heat from the gas oscillating at the ambient side of the stack) and are placed between the stack holder and the conical shape section at a distance of about $20 \mathrm{~cm}$ from the closed end of the "hot" duct. This location is around midway the velocity node and antinode and allows a wide range of velocity amplitudes (or, equivalently, gas displacement amplitudes) to be selected by varying the heat input to the system. This information has been deduced by simulating the engine behavior by highly specialized design tools of thermoacoustics systems, namely the DelatEC computer code (Design Environment for Low-Amplitude ThermoAcoustic Engines) [16], developed at the Los Alamos National Laboratory (see Section 5).

Note that the test-rig arrangement above described do not correspond to a requirement of performance optimization but, rather, to the need of guaranteeing that the HXs under test (1) be interested by substantial heat transfer rates (and associated temperature changes in the cooling water) and (2) by values of the local acoustic particle displacement ranging around (both below and above) the axial length of the HXs, as it will be more exhaustively explained in Section 5.

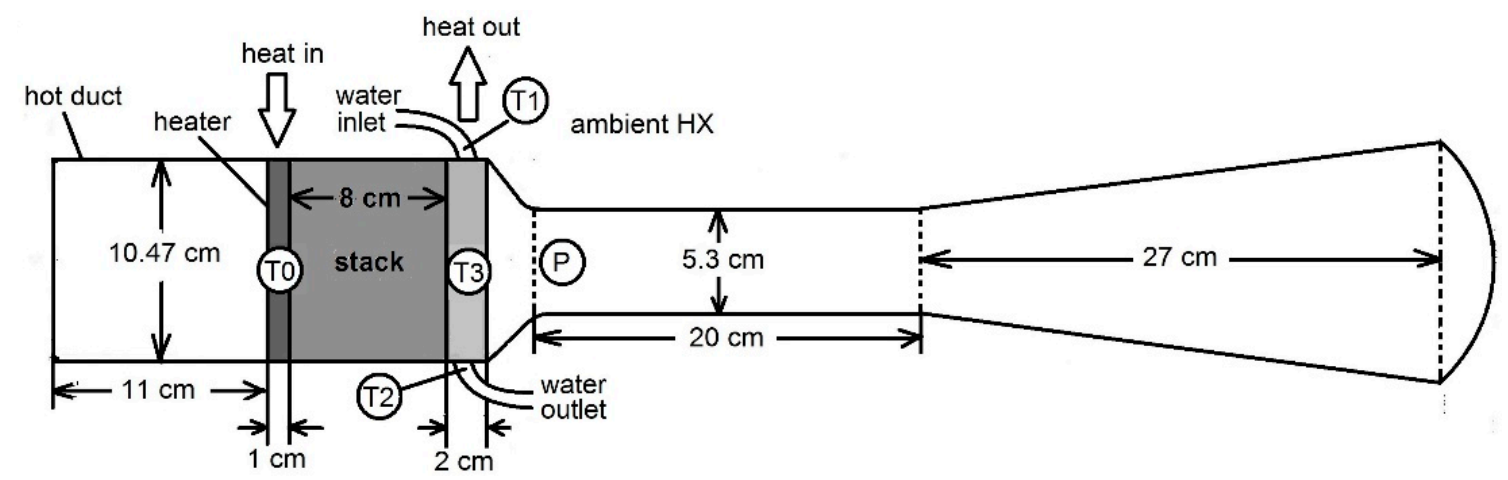

Figure 1. Schematic of the standing wave test engine. Labels " $\mathrm{T}$ " indicate thermocouple locations. Label " $\mathrm{P}$ " indicates microphone location. 


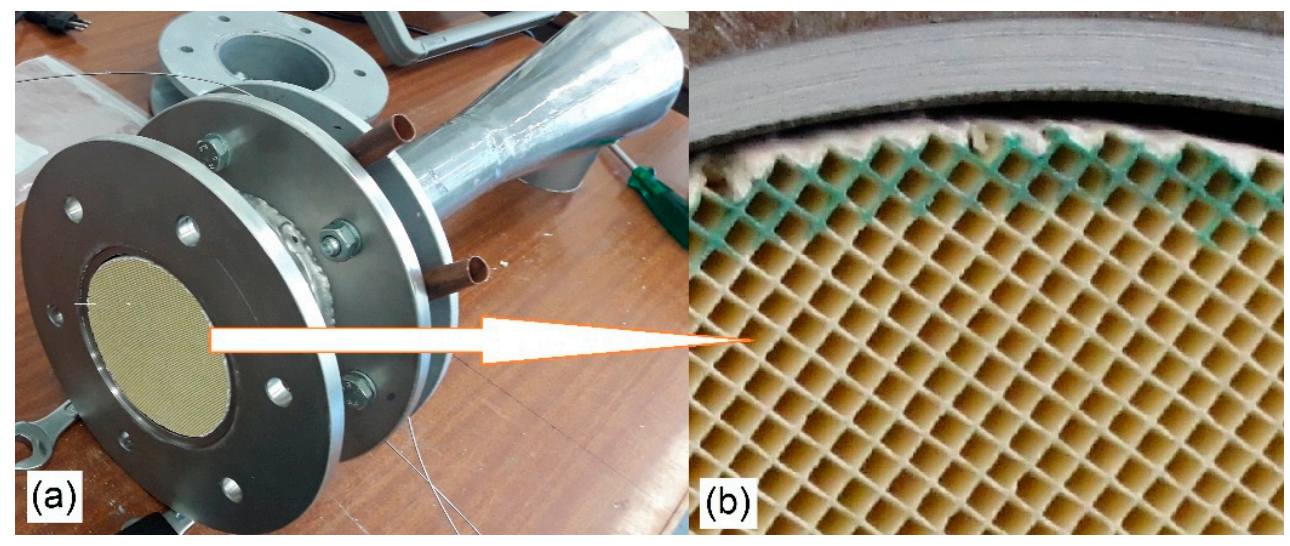

Figure 2. (a)The stack placed inside the stack-holder; (b) a magnified view of a portion of the stack.

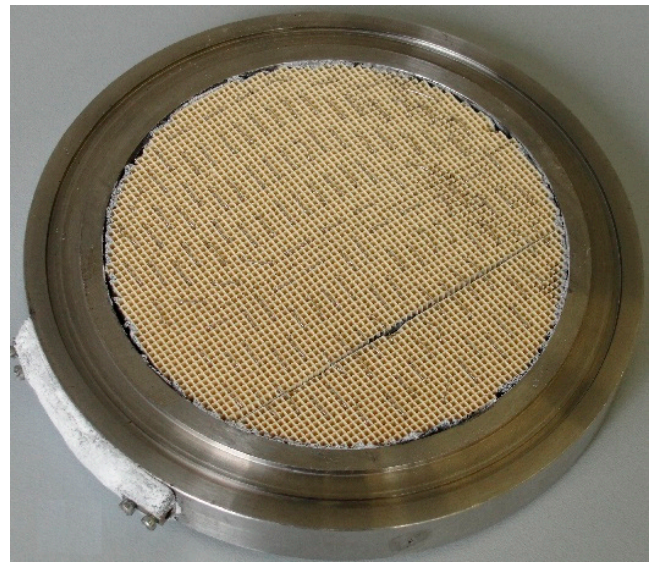

Figure 3. The heater.

\section{The Heat Exchanger Prototypes}

The HXs prototypes investigated in this study have a similar structure but differentiate each other essentially for the pore geometry. These two home-made water-cooled HXs, in fact, are both made of copper, have the same length along the resonator axis $(2 \mathrm{~cm})$, have pores of almost equal hydraulic radius and have the same flow arrangement (cross flow with four water-tube passes). The comparative test of these devices should therefore allow to study the impact of the pore geometry on the performance of the HXs and to gain information on the related gas-side heat transfer coefficient.

The first ambient HX (AHX1) has a rectangular pore geometry being of the fin-and-tube type. For its fabrication a copper ring $2 \mathrm{~cm}$ thick with an internal radius of $10.5 \mathrm{~cm}$ and an external radius of $14 \mathrm{~cm}$ has been considered as mechanical support of the HX itself. Just outside the HX the cooling water flows through a copper tube with a diameter of $14 \mathrm{~mm}$ which has been crushed to obtain a rectangular cross section pipe of area $2 \mathrm{~cm} \times 0.5 \mathrm{~cm}$. The crushed pipe has then been bent six times to allow for four passages in the front section of the HX as shown in Figure 4a. The bent segments don't obstruct the open area of the HX since they have been inserted in compartments extracted from the ring support. The fins are made of laminated copper $0.35 \mathrm{~mm}$ thick and are spaced $1 \mathrm{~mm}$ apart. They have been welded to the external walls of the pipe by a strong brazing with phosphor-copper rods. The blockage ratio $(B R)$ of this $\mathrm{HX}$, i.e., the ratio of the open to the total cross section area, is calculated to be around $55 \%$. The hydraulic radius of the pores is $R_{h}=0.5 \mathrm{~mm}$.

The second ambient heat exchanger (AXH2) is fabricated from a circular copper block. Gas passages are obtained by drilling 574 holes with a diameter of $2.5 \mathrm{~mm}$ in parallel to its axis. Cooling water passages are obtained by drilling 4 channels with a diameter of $5 \mathrm{~mm}$ perpendicularly to the 
HX axis, as shown in Figure $4 \mathrm{~b}$. The water also flows partially around the perimeter of the block. The blockage ratio of this HX is calculated to be around 33\%. The hydraulic radius of the pores is $R_{h} \approx 0.6 \mathrm{~mm}$. Compared to AHX1 this heat exchanger is characterized by a simpler fabrication technique but the circular pore geometry hardly allows to get blockage ratios exceeding $40 \%$.

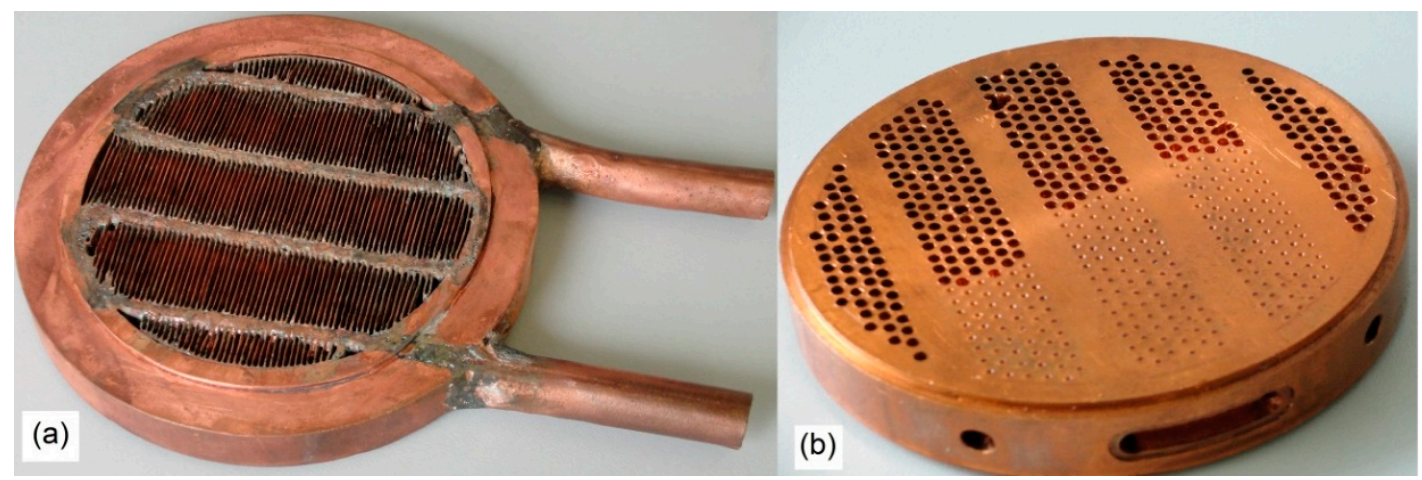

Figure 4. (a) The ambient heat exchanger with rectangular pore geometry; (b) The ambient heat exchanger with circular pore geometry.

Both HXs are characterized, as above specified, by pores of almost equal hydraulic radius $\left(R_{h}=0.5 \mathrm{~mm}\right.$ and $\left.0.6 \mathrm{~mm}\right)$. This is a precise design choice since, as inferred by simulations, at the design working frequency of the engine $(f \approx 155 \mathrm{~Hz})$ the thermal penetration depth of the gas

$$
\delta_{\kappa}=\sqrt{\frac{2 \kappa}{\omega}}
$$

i.e., the distance that heat can diffuse in an acoustic cycle, amount approximately to $\delta_{\kappa} \approx 0.22 \mathrm{~mm}$ so that $R_{h} / \delta_{\kappa} \approx 2$. This assures a good thermal contact between the oscillating gas and the HX solid walls. In Equation (1) $\kappa$ and $\omega$ are the gas thermal diffusivity and the angular frequency of the acoustic wave respectively.

A picture of the standing wave engine in place for testing without and with thermal insulation (rock wool) is shown in Figure 5a,b respectively.

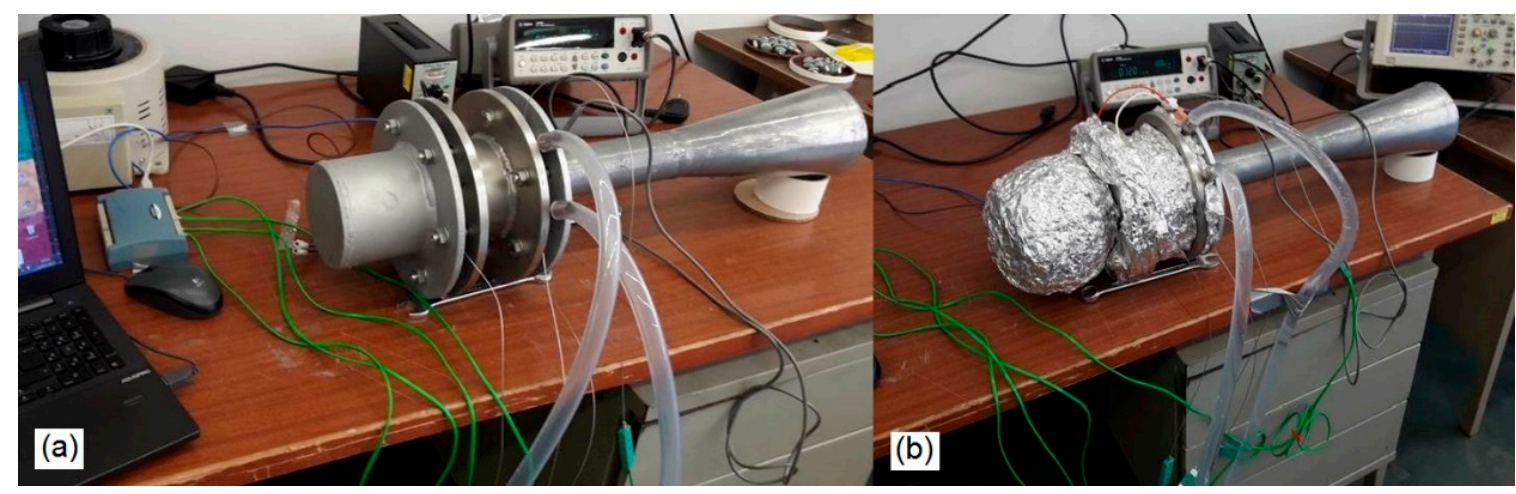

Figure 5. The standing wave test engine; (a) without thermal insulation (rock wool) and (b) with thermal insulation.

\section{The Measurement Methodology}

To get information of the heat transfer rates sustained by the HXs and on the related gas-side heat transfer coefficient experimental and calculation procedures based on standard energy balance measurement are developed. As for the first parameter, the methodology involves the determination 
of the temperature at the inlet section of the water flow $\left(T_{w i}\right)$, the one at the outlet section $\left(T_{w o}\right)$ and the mass flow rate of water $\left(\dot{m}_{w}\right)$. This allows to calculate the thermal power captured by the HX as

$$
\dot{Q}_{A}=\dot{m}_{w} c_{w}\left(T_{w o}-T_{w i}\right)
$$

where $c_{w}$ is the water specific heat. Note that the standing wave test rig discussed so far is not connected to any acoustic load. In other words, there is no component (loudspeakers, linear alternator, resistance/compliance series assembly, etc.) which could extract a fraction of the acoustic power produced by the stack from the engine. More exactly, the acoustic load is constituted by the internal surfaces of the resonator and by the HXs solid walls themselves over which the acoustic power is entirely consumed by viscous dissipation and thermal relaxation processes. Therefore, in the absence of thermal and/or acoustic power leakages to/from the outside environment, the heat extracted by the ambient HX, and represented by Equation (2), should precisely coincide with the heat delivered by the heater to the system $\left(\dot{Q}_{H}\right)$. To reduce uncertainty in measurements the system (from the "hot" duct to the AHX) is thermally insulated from the external ambient by rock wool.

To estimate the gas-side heat transfer coefficient a number of thermocouples need to be placed/embedded in the HXs with a radial distribution to measure the temporally and spatially averaged temperatures of the solid walls and of the gas locally oscillating. A mean heat transfer coefficient can be then calculated from the ratio of the heat transfer rate per unit exchange area and the temperature difference between the gas and the solid walls of the HX. For AHX1 a different procedure, involving the measurement of the temporally and spatially averaged temperature of the gas in the HX $\left(T_{g}\right)$ near the ambient side of the stack can be applied [11]. For a cross-flow heat exchanger, in fact, the heat transfer rate can be expressed as

$$
\dot{Q}_{A}=U^{\prime} A \theta_{m l},
$$

where $U^{\prime} A$ is the overall thermal conductance of the $\mathrm{HX}$ and $\theta_{m l}$, the log-mean temperature difference, is defined as

$$
\theta_{m l}=F \frac{T_{w o}-T_{w i}}{\ln \left(\frac{T_{g}-T_{w i}}{T_{g}-T_{w o}}\right)},
$$

$F$ being a correction factor for cross flow HXs and having taken into account that, since the flow is oscillatory, the inlet and outlet temperatures of the primary fluid (the gas) are the same $\left(T_{g}\right)$. Once calculated the values of $\dot{Q}_{A}$ and $\theta_{m l}$ from the experimental measurements it is possible to evaluate the overall thermal conductance of the HX from Equation (3). To estimate the gas-side heat transfer coefficient the overall conductance $U^{\prime} A$ must now be expressed in an explicit form. A simplified heat transfer model provides [17].

$$
U^{\prime} A=\frac{1}{\frac{1}{h_{w} A_{i}}+\frac{s}{K_{t} A_{i}}+\frac{1}{h\left[\sum_{i}\left(\Pi L_{f i}\right) \eta_{f i}+A_{b}\right]}},
$$

where $h_{w}$ is the water-side heat transfer coefficient, $A_{i}$ is the total inner surface of the tubes, $K_{t}$ is the thermal conductivity of the tube material (copper), $s$ is the wall thickness of the tube, $h$ is the gas-side heat transfer coefficient, $\Pi$ is the perimeter of the fin cross section, $L_{f i}$ is half the length of the $i$-th fin, $A_{b}$ is the total area of the unfinned external surface of the tubes and where $\eta_{f i}$, the efficiency of the $i$-th fin, is defined as

$$
\eta_{f i}=\frac{\tanh \left(m L_{f i}\right)}{m L_{f i}}, \quad m=\sqrt{\frac{h \Pi}{A_{f} K_{f}}}
$$

$A_{f}$ and $K_{f}$ being the cross section area and thermal conductivity of the fin respectively. In deducing Equation (5) it has been considered that the tubes for the passage of the water have a rectangular cross section so that the associated thermal resistance can be approximated by that of a plane wall. 
The heat transfer coefficients derived by means of the above method have to be correlated to the local oscillating velocity or, more exactly, to the acoustic Reynolds number $\operatorname{Re}_{1}=\rho_{0} D_{h} v / \eta$ ( $\rho_{0}$ being the mean gas density, $D_{h}$ the hydraulic diameter of the HX pore, $v$ the acoustic velocity amplitude and $\eta$ the dynamic viscosity). This last is derived by modelling the standing-wave engine by the DeltaEC code and evaluating the acoustic particle velocity at the position of the ambient $\mathrm{HX}$. The validation of the data involves an iterative refinement of the model by the acoustic pressure values measured in different positions along the resonator axis by dynamic pressure sensors.

\section{Simulations with DeltaEC}

To model the thermoacoustic engine and get information on the acoustic field neat the test HXs the computer code DeltaEC has been used. The code integrates the linear theory of thermoacoustics, firstly formulated by Rott [18] and subsequently refined by Swift [1,2], which has demonstrated accurate precision for pressure amplitudes up to $10 \%$ of the mean pressure. The code performs 1D numerical integration of the momentum, continuity and energy equations through each segment of the acoustic network. The solutions found for adjoining segments are then matched by imposing the continuity of pressure and volume flow rate at their junction. For further details the reader is addressed to ref. [16].

In Figure 6 the axial distribution of the acoustic field (acoustic pressure, $p$, and volumetric velocity, $U$ ) is shown. The $p$ and $U$ profiles resemble those characterizing a half-wavelength resonator. The graph shows how the HX location falls between a velocity node and antinode. The acoustic particle velocity near the HX can be modulated by varying the heat input to the system. This also influences the operative temperature of the heater, $T_{H}$, the acoustic pressure amplitude, $p$, and the amplitude of the particle oscillation displacement

$$
x_{1}=\frac{U}{A_{H X} \omega}
$$

$A_{H X}$ being the cross section area of the HX open to gas flow. The last is an important parameter in the design of HXs since, being the flow oscillatory, an excessively long HX (along the longitudinal direction of the particle acoustic oscillation) could only lead to additional thermoviscous losses without incrementing the surface area available for heat transfer.

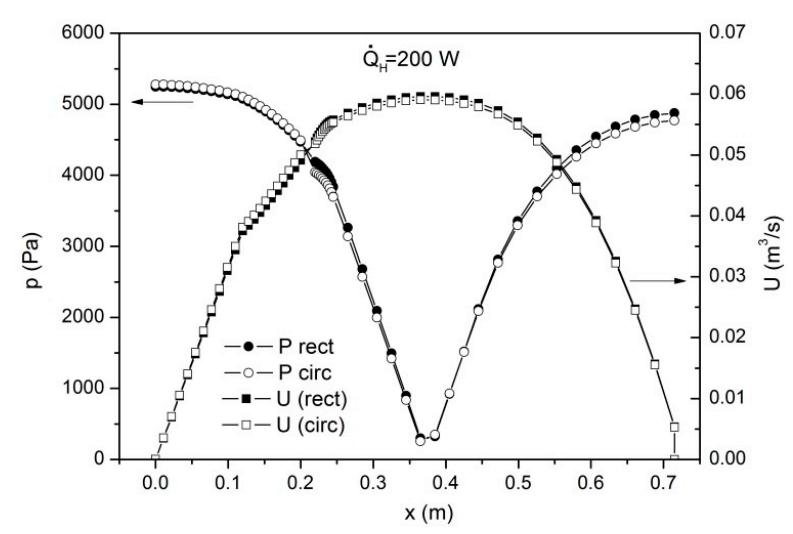

Figure 6. The axial distribution of the acoustic pressure amplitude and volumetric velocity amplitude.

Conversely, a much too short HX could be ineffective in transferring the required thermal load. It is generally assumed, as a rule of tumb, that the optimal length of the HX $\left(L_{H X}\right)$ should be of the order of the peak-to-peak acoustic displacement amplitude [1], although this problem remains to date an open issue.

In Figure 7 the variables $T_{H}, D R$ and $2 x_{1} / L_{H X}$ are reported as a function of the heat input for the two typologies of HX. In both cases the drive ratio is lower than $10 \%$ even at the highest power delivered by the heater so the growth of non linear acoustic effects should not considerably affect the engine operation. But, more interesting, is the circumstance that at a given $\dot{Q}_{H}$ value the 
peak-to-peak particle displacement amplitude, $2 x_{1}$, surpasses the length of the HX. This "transition" should allow to investigate on the optimal length along the longitudinal direction which maximizes the HX performance.

As a concluding remark it has to be underlined that DeltaEC relies, as for the calculation of the gas side heat transfer coefficient, on a simple boundary layer model expressed by the equation

$$
h=\frac{K}{y_{e f f}}
$$

where

$$
y_{\text {eff }}=\min \left\{\delta_{\kappa}, R_{h}\right\},
$$

It is retained that this expression may be quite inaccurate and be affected by an error of about a factor of two.
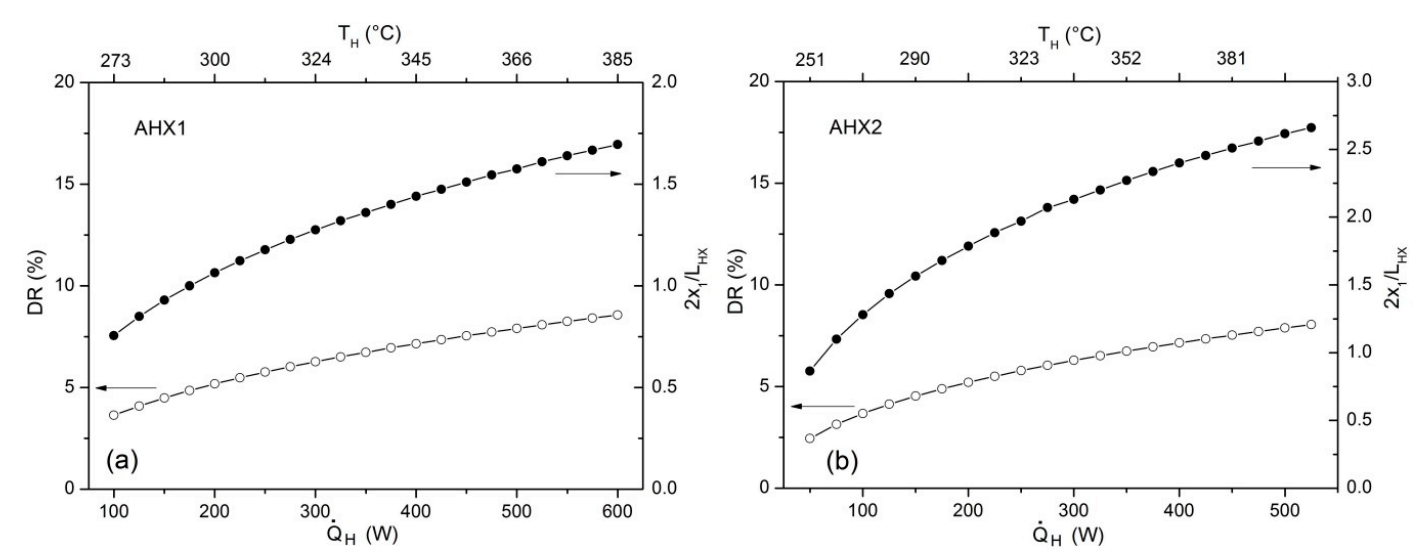

Figure 7. The drive ratio and peak-to-peak particle displacement amplitude as a function of the input heat flux for (a) the HX with rectangular pore geometry and (b) the HX with circular pore geometry. The operation temperatures of the heater, $T_{H}$, are also indicate on the horizontal scale on the top.

\section{Preliminary Experimental Results}

In Figures 8 and 9 some preliminary experimental results concerning AHX1 are shown. In the first graph the difference between the temperatures of the hot HX (the heater) and of the ambient HX is reported as a function of the measured input heat transfer rate $\left(\dot{Q}_{H}\right)$. The thermocouple (K-type thermocouple) of the heater was placed inside a pore of the ceramic honeycomb support so it presumably measured a mean value $\left(T_{H}\right)$ between the gas and the solid wall temperatures.

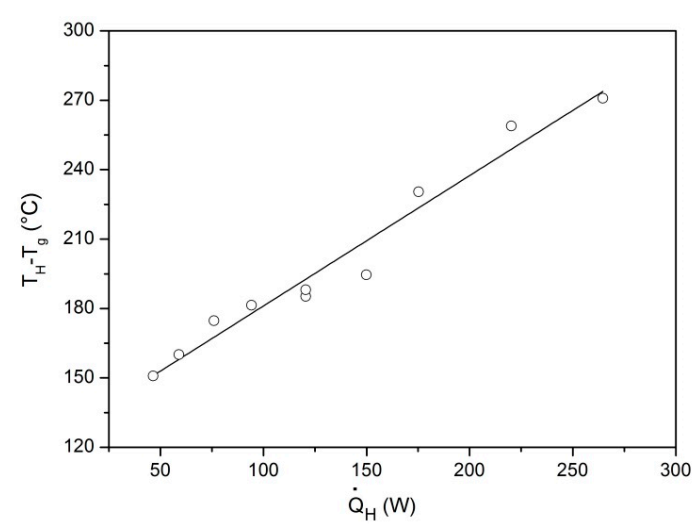

Figure 8. The temperature difference between the hot and ambient heat exchanger with rectangular pores as a function of the input heat. 
The thermocouple of the ambient HX was suspended by a little spacer in the gap between two fins so in this case the gas temperature $\left(T_{g}\right)$ was measured. The input heat flux $\dot{Q}_{H}$ was calculated by measuring the voltage drop across the heater and the flowing current. The heat flux extracted by the ambient $H X, \dot{Q}_{A}$, was calculated through Equation (2) from the measured values of water mass flow rate $\left(\dot{m}_{w} \approx 0.052 \mathrm{~kg} / \mathrm{s}\right)$ and of its temperature at the inlet $\left(T_{w i} \approx 300 \mathrm{~K}\right)$ and outlet sections of the HX measured by two thermocouples. The acoustic pressure values reported in Figure 9 were measured by a condenser microphone (sensitivity $1.589 \mathrm{mV} / \mathrm{Pa}$ ) flush mounted in the wall of the narrow duct at a distance of $3 \mathrm{~cm}$ from the ambient HX (see Figure 1).

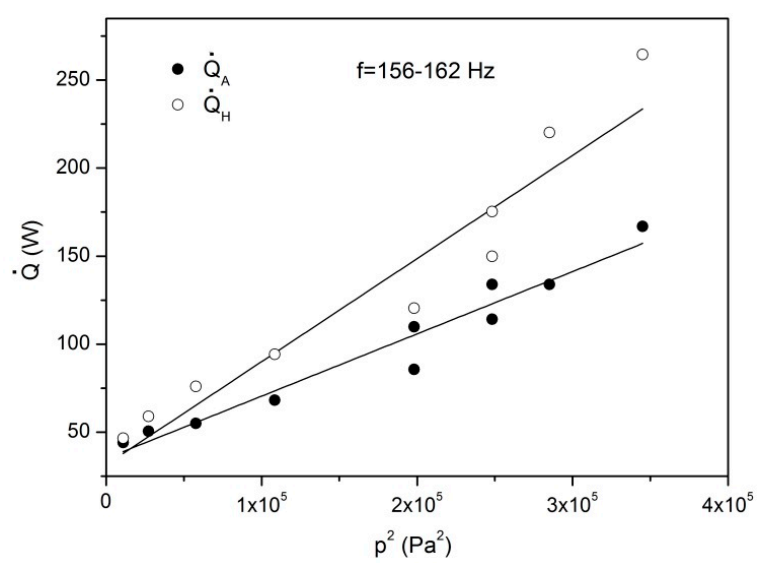

Figure 9. The heat introduced by the heater and extracted by the ambient heat exchanger with rectangular pores as a function of the square of the acoustic pressure.

The air starts to oscillate spontaneously in the engine for $T_{H} \approx 200{ }^{\circ} \mathrm{C}$ at a (resonance) frequency of $156.5 \mathrm{~Hz}$ (which slightly grew with $\left.\dot{Q}_{H}\right)$. Figure 8 shows how the temperature difference $\left(T_{H}-T_{g}\right)$ grows quickly and almost linearly with $\dot{Q}_{H}$ in the investigated range. A linear behavior is also found in Figure 9 where $\dot{Q}_{H}$ and $\dot{Q}_{A}$ are reported as a function of the square of the acoustic pressure. Note, however, as the difference between $\dot{Q}_{H}$ and $\dot{Q}_{A}$ (increasing with the heater power) is a clear evidence of heat and/or acoustic power leakage toward the external environment, so an improvement of the engine insulation is required.

These findings are in accordance with the linear theory of thermoacoustics and let suppose that in the investigated operation range non linear effects are negligible. This result is somehow expected when considering that at the highest $\dot{Q}_{H}$ values shown in Figure 9 the measured acoustic pressure reached $0.6 \%$ of the mean pressure. Since the microphone is located around halfway a pressure node and antinode this value should roughly correspond to a drive ratio of about $1.2 \%$, that is well below the onset level of non linear effects.

\section{Conclusions}

The research described in the present paper focuses on the development of experimental techniques for investigating on the fundamental issue of heat transfer of thermoacoustic heat exchangers under acoustically oscillating flows. The test rig, a standing wave engine working with air at atmospheric pressure, and the test heat exchangers, a circular pore and rectangular pore cross flow heat exchanger, have been designed and manufactured. The prototype has been conceived to study the performance of the heat exchangers under a range of operating conditions (drive ratio, operation frequency, acoustic particle velocity temperature differences, etc.) and to estimate, by specific procedures, the magnitude of gas side heat transfer coefficient relatively to the two considered pore geometries. The present research, the initial stage of which is discussed in this paper, could help in achieving a deeper understanding of the heat transfer processes affecting heat exchangers 
under oscillating flow regime and in developing optimization procedures of their performance in the design phase.

Acknowledgments: This research did not receive any specific grant from funding agencies in the public, commercial, or not-for-profit sectors. The authors are grateful to the reviewers for their suggestions which largely contributed to improve the quality of the present paper.

Author Contributions: Antonio Piccolo conceived and designed the experiments, built the heater and the HX with circular pores, performed the experiments and numerical simulations, analysed the data and wrote the paper; Roberto Siclari built the resonator and the HX with parallel fins and contributed to analyse the data; Fabrizio Rando contributed to perform the experiments and analyse the data; Mauro Cannistraro contributed to analyse the data and write the paper.

Conflicts of Interest: The authors declare no conflict of interest.

\section{References}

1. Swift, G.W. Thermoacoustic engines. J. Acoust. Soc. Am. 1988, 84, 1145-1180.

2. Swift, G.W. Thermoacoustics: A unifying perspective for some engines and refrigerators. J. Acoust. Soc. Am. 2002, 113. [CrossRef]

3. Backhaus, S.; Tward, E.; Petach, M. Traveling-wave thermoacoustic electric generator. Appl. Phys. Lett. 2004, 85, 1085-1087. [CrossRef]

4. Yu, Z.; Jaworski, A.J.; Backhaus, S. Travelling-wave thermoacoustic electricity generator using an ultra-compliant alternator for utilization of low-grade thermal energy. Appl. Energy 2012, 99, 135-145. [CrossRef]

5. Piccolo, A. Design issues and performance analysis of a two-stage standing wave thermoacoustic electricity generator. Sustain. Energy Technol. Assess. 2016, in press. [CrossRef]

6. Backaus, S.; Swift, G.W. A thermoacoustic Stirling heat engine: Detailed study. J. Acoust. Soc. Am. 2000, 107, 3148-3166. [CrossRef]

7. Swift, G.W. Analysis and performance of a large thermoacoustic engine. J. Acoust. Soc. Am. 1992, 92, 1551-1563. [CrossRef]

8. Garrett, S.L.; Perkins, D.K.; Gopinath, A. Thermoacoustic Refrigerator Heat Exchangers: Design, Analysis and Fabrication. In Proceedings of the Tenth International Heat Transfer Conference, Brighton, UK, 14-18 August 1994; pp. 375-380.

9. Piccolo, A. Numerical study of entropy generation within thermoacoustic heat exchangers with plane fins. Entropy 2015, 17, 8228-8239. [CrossRef]

10. Piccolo, A. Optimization of thermoacoustic refrigerators using second law analysis. Appl. Energy 2013, 103, 358-367. [CrossRef]

11. Peak, I.; Braun, J.E.; Mongeau, L. Characterizing heat transfer coefficients for heat exchangers in standing wave thermoacoustic coolers. J. Acoust. Soc. Am. 2005, 118, 2271-2280. [CrossRef]

12. Jaworski, A.J.; Piccolo, A. Heat transfer processes in parallel-plate heat exchangers of thermoacoustic devices-Numerical and experimental approaches. Appl. Therm. Eng. 2012, 42, 145-153. [CrossRef]

13. Mohd Saat, F.A.Z.; Jaworski, A.J. The effect of temperature field on low amplitude oscillatory flow within a parallel-plate heat exchanger in a standing wave thermoacoustic system. Appl. Sci. 2017, 7, 417. [CrossRef]

14. Tijani, M.E.H.; Zeegers, J.C.H.; De Waele, A.T.A.M. Design of thermoacoustic refrigerators. Cryogenics 2002, 42, 49-57. [CrossRef]

15. Piccolo, A.; Pistone, G. Computation of the time-averaged temperature fields and energy fluxes in a thermally isolated thermoacoustic stack at low acoustic Mach numbers. Int. J. Therm. Sci. 2007, 46, 235-244. [CrossRef]

16. Clark, J.P.; Ward, W.C.; Swift, G.W. Design environment for low-amplitude thermoacoustic energy conversion (DeltaEC). J. Acoust. Soc. Am. 2007, 122. [CrossRef]

17. Incoprera, F.P.; De Witt, D.P. Fundamentals of Heat and Mass Transfer, 3rd ed.; Wiley and Sons: New York, NY, USA, 1996.

18. Rott, N. Thermoacoustics. Adv. Appl. Mech. 1980, 20, 35-75.

(C) 2017 by the authors. Licensee MDPI, Basel, Switzerland. This article is an open access article distributed under the terms and conditions of the Creative Commons Attribution (CC BY) license (http:// creativecommons.org/licenses/by/4.0/). 